\title{
Correction to: A computational model of a network of initial lymphatics and pre-collectors with permeable interstitium
}

\author{
B. O. Ikhimwin ${ }^{1} \cdot$ C. D. Bertram ${ }^{1} \cdot$ S. Jamalian ${ }^{2} \cdot$ C. Macaskill $^{1}$
}

Published online: 18 February 2020

๑) Springer-Verlag GmbH Germany, part of Springer Nature 2020

\section{Correction to: Biomechanics and Modeling in Mechanobiology https://doi.org/10.1007/s10237-019-01238-x}

In the published paper (Ikhimwin et al. 2019), we argued that, although there appear to be no data available on the subject, it is inherently unlikely that lymph having traversed a network of initial lymphatics and pre-collectors then encounters an abrupt transition to vessels with all of the typical properties of collecting lymphatics (relatively thick muscular wall, endothelial cells with tight junctions). Rather, we argued for a gradual spatial transition from vessels with maximal primary-valve availability to ones with none. As one of our parameter variations, we therefore chose to create a model with primary-valve availability tapering away gradually in the streamwise direction. In our models, the hydraulic resistance to inflow through the wall via the primary valves ultimately resolves to a single quantity for each numerical vessel segment. We created a model with reduced primary-valve availability downstream by multiplying each such resistance by the generation number of the vessel, where our network of 19 anatomical segments consisted of seven generations. Thus, the resistance to fluid inflow through the wall of the outflow trunk for the whole network of initial lymphatics and pre-collectors was multiplied by seven, relative to the resistances calculated for twigs in generation one. In our equations, this multiplication was reflected by a factor $G$ in the numerator of eq. 8 .

The original article can be found online at https://doi.org/10.1007/ s10237-019-01238-x.

$\triangle$ C. D. Bertram

c.bertram@sydney.edu.au

1 School of Mathematics and Statistics, University of Sydney, Sydney, NSW, Australia

2 Department of Biomedical Engineering, Johns Hopkins University School of Medicine, Baltimore, MD, USA

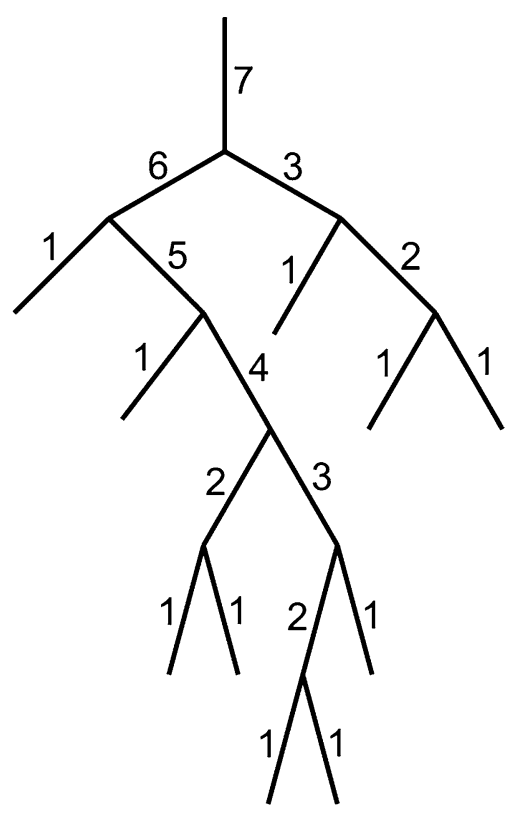

Figure C1 The 19-segment network, with Horsfield generation numbers.

However, the scheme we used to number the generations, as shown in Fig. 3 of the paper, was sub-optimal; generations were numbered according to the row in which they appeared in the network schematic. This meant that the entry twigs into the network were not all in generation one; such twigs carried generation numbers as high as five.

We have now corrected this oversight, using instead generation numbers organized according to the scheme devised by Horsfield (1976). There are still seven generations in the network, but all twigs are now in generation one, as shown in Figure $\mathrm{C} 1$. This is a much more appropriate basis on which to vary the availability (or area density) of primary valves with streamwise position within the network.

This change affects the results which are presented in Figs. 5, 6 and 7 of the published paper. We accordingly here 
Figure C2 Equivalent to Fig. 5 in the published paper. External pressure, outlet diameter and outlet flow rate versus time for the network with spatially uniform (red and blue curves) or tapering (green and grey curves) PV density, and nonleaky (red and green curves) or leaky (blue and grey curves) PVs. The period of oscillation is $2 \mathrm{~s}, p_{\text {out }}=1.4 \mathrm{~cm} \mathrm{H}_{2} \mathrm{O}$ (magenta line), and $p_{\mathrm{e}}(t)$ varies between 1 and $2 \mathrm{cmH}_{2} \mathrm{O}$ with mean $1.5 \mathrm{~cm} \mathrm{H}_{2} \mathrm{O}, r_{\mathrm{is}}=300 \mu \mathrm{m}$, $m=2.1 \times 10^{5}$ dyn $\mathrm{cm}^{-3}$ and $d_{\mathrm{ec}}=2 \mu \mathrm{m}$. Solid vertical lines indicate when $p_{\mathrm{e}}(t)$ crosses $p_{\text {out }}$. Dashed vertical lines indicate when $Q_{\text {out }}(t)$ crosses zero.
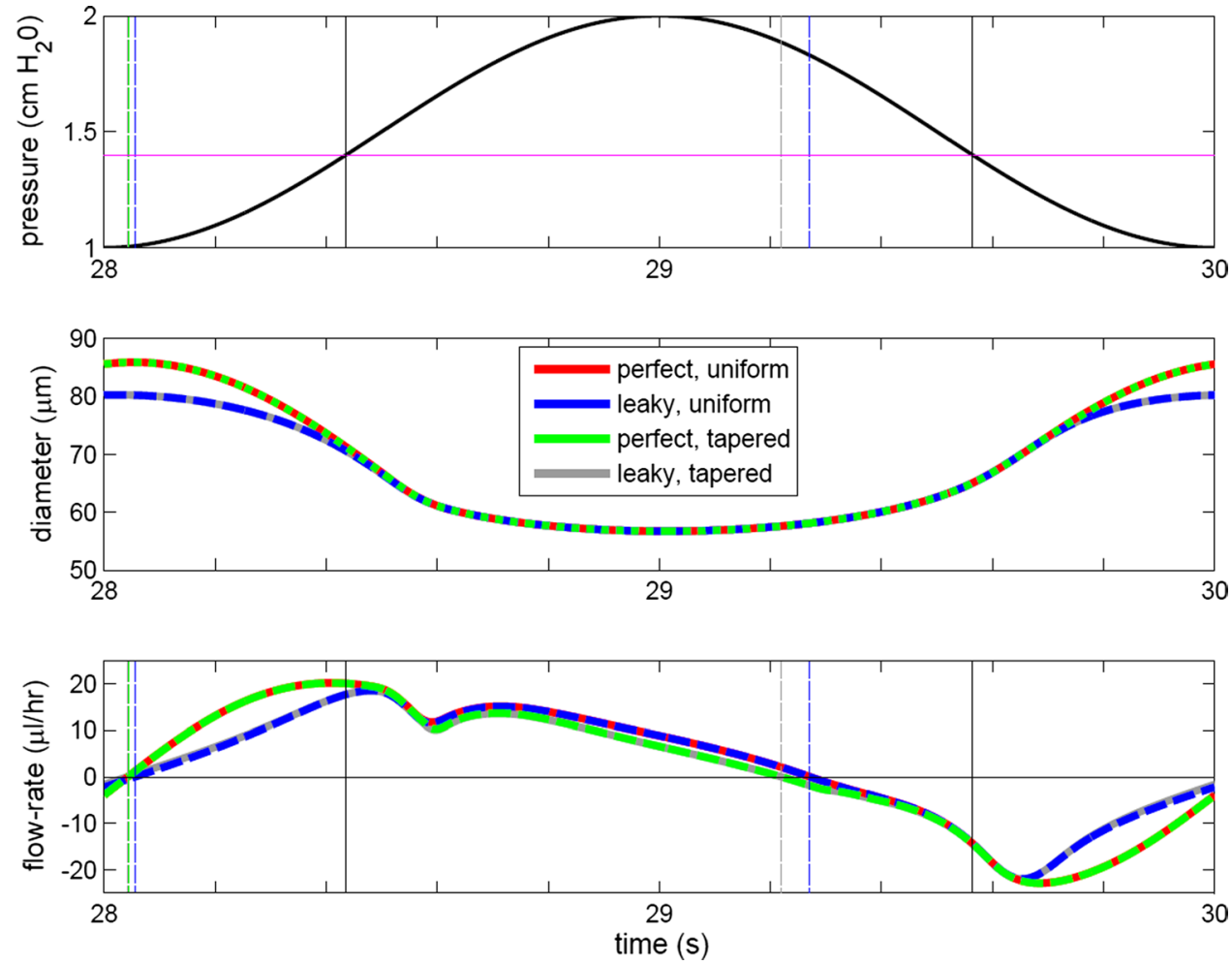

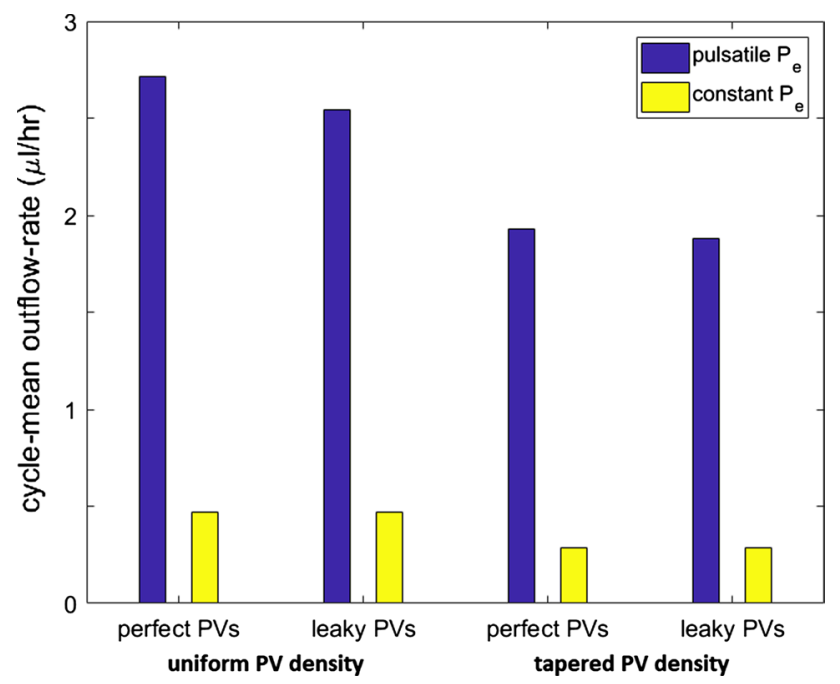

Figure C3 Equivalent to Fig. 6 in the published paper. Comparison of $Q_{\text {cyc-av }}$ in the outlet branch when $p_{\mathrm{e}}$ varied between 1 and $2 \mathrm{~cm} \mathrm{H}_{2} \mathrm{O}$ with mean $1.5 \mathrm{~cm} \mathrm{H}_{2} \mathrm{O}$ (blue bars), with the steady flow rate when $p_{\mathrm{e}}$ was constant at $1.5 \mathrm{~cm} \mathrm{H}_{2} \mathrm{O}$ (yellow bars), for both uniform and spatially tapering PV function, and both non-leaky and leaky PVs. Parameters: $p_{\text {out }}=1.4 \mathrm{~cm} \mathrm{H}_{2} \mathrm{O}, r_{\text {is }}=300 \mu \mathrm{m}, m=2.1 \times 10^{5} \mathrm{dyn}^{-3}$, $d_{\mathrm{ec}}=2 \mu \mathrm{m}$.

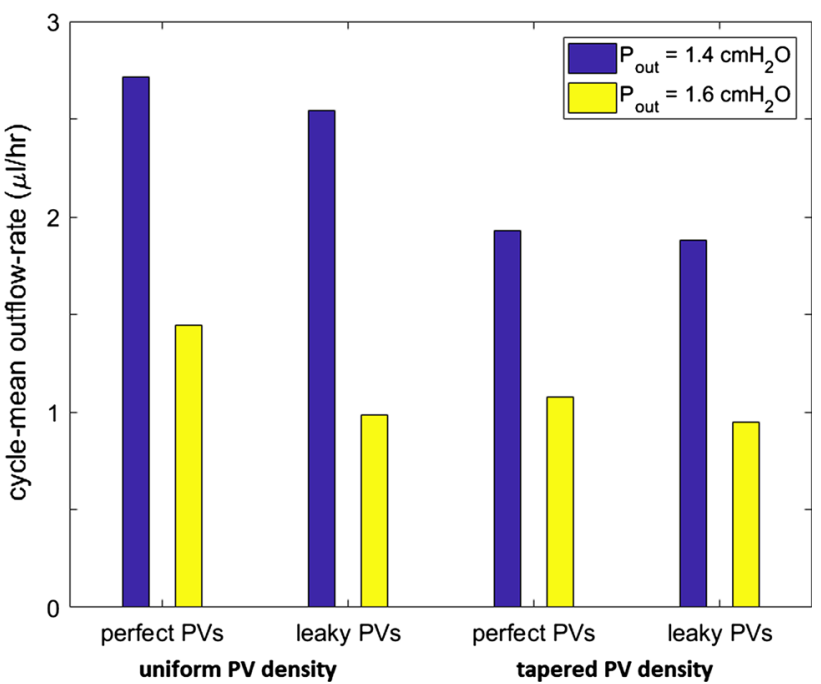

Figure C4 Equivalent to Fig. 7 in the published paper. Comparison of $Q_{\text {cyc-av }}$ exiting the network between $p_{\text {out }}<p_{\mathrm{e}}$ (blue bars) and $p_{\text {out }}>p_{\mathrm{e}}$ (yellow bars) for uniform and spatially tapering PV function. External pressure varied between 1 and $2 \mathrm{~cm} \mathrm{H}_{2} \mathrm{O}$ with mean $1.5 \mathrm{~cm} \mathrm{H}_{2} \mathrm{O}$, for both non-leaky and leaky PVs. Parameters: $r_{\text {is }}=300 \mu \mathrm{m}, m=2.1 \times 10^{5}$ dyn $\mathrm{cm}^{-3}, d_{\mathrm{ec}}=2 \mu \mathrm{m}$ 
provide replacement versions of these figures, in which the Horsfield generation number has been used to calculate all results involving tapered primary-valve density, i.e. $H$ has replaced $G$ in Eq. 8 .

\section{References}

Horsfield K (1976) Some mathematical properties of branching trees with application to the respiratory system. Bull Math Biol 38:305-315
Ikhimwin BO, Bertram CD, Jamalian S, Macaskill C (2019) A computational model of a network of initial lymphatics and pre-collectors with permeable interstitium. Biomech Model Mechanobiol. https://doi.org/10.1007/s10237-019-01238-x

Publisher's Note Springer Nature remains neutral with regard to jurisdictional claims in published maps and institutional affiliations. 